\title{
Control of chaos in multimode solid state lasers by the use of small periodic perturbations
}

\author{
Pere Colet ${ }^{*}$ \\ Departament de Física, Universitat de les Illes Balers, E-07071 Palma de Mallorca, Spain \\ Y. Braiman \\ Department of Physics, Emory University, Atlanta, Georgia 30322
}

(Received 14 August 1995)

\begin{abstract}
We present a numerical study of control of chaos in a model of a multimode neodymium-doped yttrium aluminum garnet laser using periodic perturbations of accessible control parameters. We show that a small modulation of either the losses or the pump can eliminate chaos or make the system even more chaotic. Depending on the parameters of the perturbation, two qualitatively different periodic outputs can be achieved.
\end{abstract}

PACS number(s): 05.45. $+\mathrm{b}$, 42.65.Sf

\section{INTRODUCTION}

A great deal of attention has been paid in recent years to developing algorithms to convert dynamical systems from chaotic to periodic motion. (For comprehensive reviews, see, for example, Refs. [1].) The algorithms are based on the fact that small changes applied to a chaotic system can suppress and eliminate chaos. Moreover, these methods make it possible to settle a given dynamical system on its initially unstable orbit. The practical importance of controlling chaos is significant in many fields. Several experiments have been recently performed using the algorithms to control chaos in various physical, chemical, and biological systems. These include control of chaos in a magnetic ribbon [2], spin wave instabilities [3], Chua's circuit [4], fluids [5], laser systems, either single mode [6] or multimode [7,8], fast diode resonators [9], chemical systems [10], thin circular yttrium iron garnet film [11], and control of chaos for communication [12]. Biological applications include control of chaos in neural networks [13], in the cardiac system [14], and in the brain [15].

The different techniques in controlling chaos can be (mainly) characterized by two categories: feedback and nonfeedback. The feedback techniques are very powerful and have proven to be very efficient; they allow one to target the nonlinear dynamical system to the desired trajectory. The most used feedback techniques are the OGY control [16], the OPF technique [17], self-controlled feedback [18], and delay coordinates [19]. The nonfeedback techniques have not been so actively studied, and are less understood and more challenging. The essential advantage of nonfeedback techniques lies in their speed; no online monitoring and processing is required. This speed makes them especially promising for controlling systems

\footnotetext{
"Present address: Instituto de Estructura de la Materia, CSIC, Serrano 123, E-28006 Madrid, Spain.
}

such as fast electro-optical systems or superconducting Josephson junction arrays. Different mechanisms of nonfeedback control have been proposed: entrainment to goal dynamics (open-loop control) [20], the parametric excitation of an experimentally adjustable parameter [21], and taming the chaotic (or neutrally stable) system by the means of external periodic perturbation [22,23].

In this work we study control of chaos in a multimode neodymium-doped yttrium aluminum garnet (Nd:YAG) solid state laser with an intracavity frequency doubling potassium titanyl phosphate (KTP) crystal using a small periodic parameter modulation. The Nd:YAG laser is important in a variety of applications [24], its parametrization is well defined experimentally [25], and the laser is an example of a system with many degrees of freedom (control of chaos in extended systems is of recent interest [26]). The system is autonomously chaotic and to achieve stabilization we perform amplitude modulations of either losses or pumping rate. The modulation of losses is a parametric modulation, while modulating the pump is an example of an external modulation. We find that loss modulation is a very effective way to achieve stabilization, even for very small amplitude modulations. Pump modulations stabilize periodic orbits as well but (for parameter values we use) the amplitudes required are larger. Two qualitatively different kinds of periodic orbits can be obtained: the "smooth" orbits, where the intensities of the modes show a continuous oscillation pattern, and the "spiky" pulsed periodic output, where the intensity of the modes shows large amplitude pulses and (practically) vanishes between the pulses.

It has been shown experimentally by Meucci et al. [8] that small periodic perturbations can stabilize a singlemode $\mathrm{CO}_{2}$ laser. Chaos is induced by modulating the cavity losses using an intracavity electro-optic modulator driven by a dc plus ac voltage. Stabilization is achieved by applying a small sinusoidal modulation to the amplitude of the ac part of the voltage. More recently it has been shown that the chaotic output of a model for a semiconductor laser diode with external feedback can be stabilized by applying a sinusoidal modulated injection 
current $[27,28]$.

This paper is organized in the following way. We first introduce the model of a multimode solid state laser in Sec. II. In Sec. III we show how control of chaos can be achieved for a small periodic modulation of the losses. Section IV is devoted to stabilization using pump modulation. Concluding remarks are given in Sec. V.

\section{MULTIMODE LASER MODEL}

We consider a model of a multimode neodynium doped yttrium aluminum garnet (Nd:YAG) laser with an intracavity potassium titanyl phosphate (KTP) frequency doubling crystal. The dynamics can be described in terms of the rate equations for the intensity $I_{k}$ and gain $G_{k}$ associated with each mode [25],

$$
\begin{aligned}
& \frac{d I_{k}}{d t}=\frac{1}{\tau_{c}}\left[G_{k}-\alpha-g \epsilon I_{k}-2 \epsilon \sum_{j \neq k}^{N} \mu_{k j} I_{j}\right] I_{k}, \\
& \frac{d G_{k}}{d t}=\frac{1}{\tau_{f}}\left[p-\left(1+I_{k}+\beta \sum_{j \neq k} I_{j}\right] G_{k}\right],
\end{aligned}
$$

where $N$ is the number of modes and $k=1, \ldots, N$. Here $\tau_{c}$ is the cavity roundtrip time $(0.24 \mathrm{~ns}), \tau_{f}$ is the fluorescence lifetime of the $\mathrm{Nd}^{3+}$ ion $(240 \mu \mathrm{s}), \alpha$ is the cavity loss parameter (assumed to be the same for all the modes, $\alpha=0.01$ ), $p$ is the small signal gain, which is related to the pump parameter, and $g$ is a geometrical factor that is dependent on the phase delays due to the YAG and the KTP crystals and on the angle between the YAG and KTP fast axes; $\epsilon$ is the nonlinear coefficient associated with the conversion efficiency of the fundamental intensity into frequency doubled intensity by the KTP crystal $\left(\epsilon=5 \times 10^{-6}\right) ; \beta$ is the cross-saturation parameter related to the competition among the different longitudinal modes. In general its value will be different for each pair of cavity modes, but for simplicity we assume the same value of $\beta$ for all the mode pairs. The coefficients $\mu_{j k}$ depend on the mode polarization. Each cavity mode can be polarized only in one of two orthogonal directions: $\mu_{j k}=g$ if modes $j$ and $k$ have the same polarization, and $\mu_{j k}=1-g$ if modes $j$ and $k$ have orthogonal polarizations.

Here we consider the case of three modes, two polarized in one direction, and one in the orthogonal direction. It is convenient to rescale the time as $s=t / \tau_{f}$, so that the set of Eqs. (1) can be rewritten as

$$
\begin{aligned}
& \dot{I}_{k}=\tau\left[G_{k}-\alpha-g \epsilon I_{k}-2 \epsilon \sum_{j \neq k}^{N} \mu_{k j} I_{j}\right] I_{k}, \\
& \dot{G}_{k}=p-\left[1+I_{k}+\beta \sum_{j \neq k} I_{j}\right] G_{k},
\end{aligned}
$$

where the differentiation is with respect to $s$ and $\tau=\tau_{f} / \tau_{c}$. This set of equations is symmetric for modes 1 and 2 , which both have the same polarization. Assume we keep all the parameters fixed, increasing the pumping $p$; then the system evolves from a stable steady state to periodic, and finally to chaotic behavior.

\section{TAMING CHAOS WITH A SMALL PERIODIC MODULATION OF THE LOSSES}

In this section we study the effect of applying a small periodic modulation of the losses on a multimode Nd:YAG laser with an intracavity KTP frequency doubling crystal in the regime where without modulation the laser is chaotic. A typical chaotic time trace is shown in Fig. 1. The losses are modulated in the following way:

$$
\alpha=\alpha_{0}\left[1+\alpha_{1} \sin (\omega s)\right],
$$

where $\alpha_{0}=0.01$ and the dimensionless modulation frequency $\omega$ is defined as $\omega=\Omega \tau_{f}, \Omega$ being the modulation frequency.

The numerical simulations show that a very small modulation applied to the losses leads to a large dynamical effect on the system. Depending on the modulation amplitude and the modulation frequency, it can either eliminate chaos or even significantly increase it. Figure 2 shows the values of the leading Liapunov exponent as a function of loss modulation amplitude for three different modulation frequencies: $\omega=40 \quad\left(\Omega=166.67 \mathrm{~ms}^{-1}\right)$, $\omega=50 \quad\left(\Omega=208.33 \mathrm{~ms}^{-1}\right)$, and $\omega=100 \quad(\Omega=416.67$ $\left.\mathrm{ms}^{-1}\right)$. In all three cases stabilization is possible for very small values of the modulation amplitude. Starting from zero modulation amplitude, the general trend of the value of the leading Liapunov exponent is to decrease as the modulation amplitude increases, reaching a negative value, so that the system is not chaotic anymore. Figure 3 shows the time trace for the intensities of three modes when the system is stabilized with $\omega=40$ and $\alpha_{1}=0.025$. The oscillations of modes 1 and 2 are synchronized, while mode 3 reaches its maximum value when the other two are small. It is also possible to stabilize periodic orbits where modes 1 and 2 oscillate in an asymmetric way, as shown in Fig. 4 for $\omega=100$ and $\alpha_{1}=0.006$.

For larger modulation amplitudes, the leading

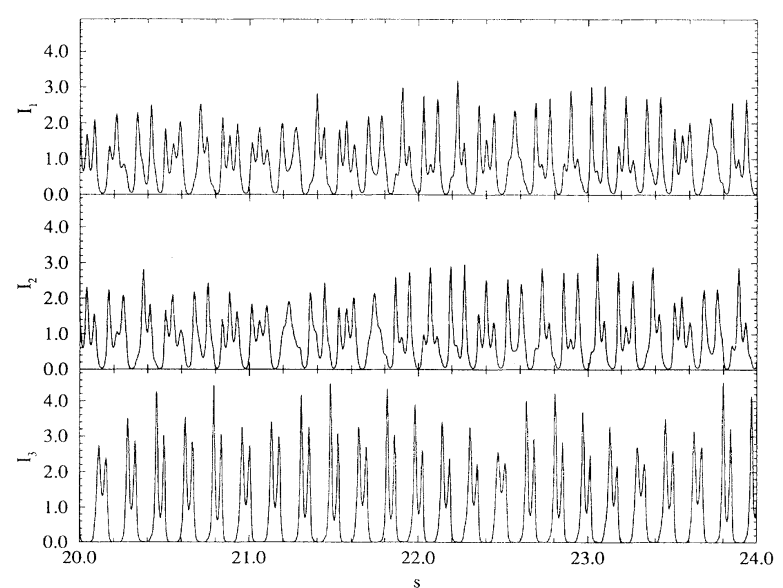

FIG. 1. Typical chaotic time trace obtained from numerical integration of the set of equations (2) for $g=0.1, \beta=0.65$, and $p=0.03$. The quantities plotted in all the figures are dimensionless. 

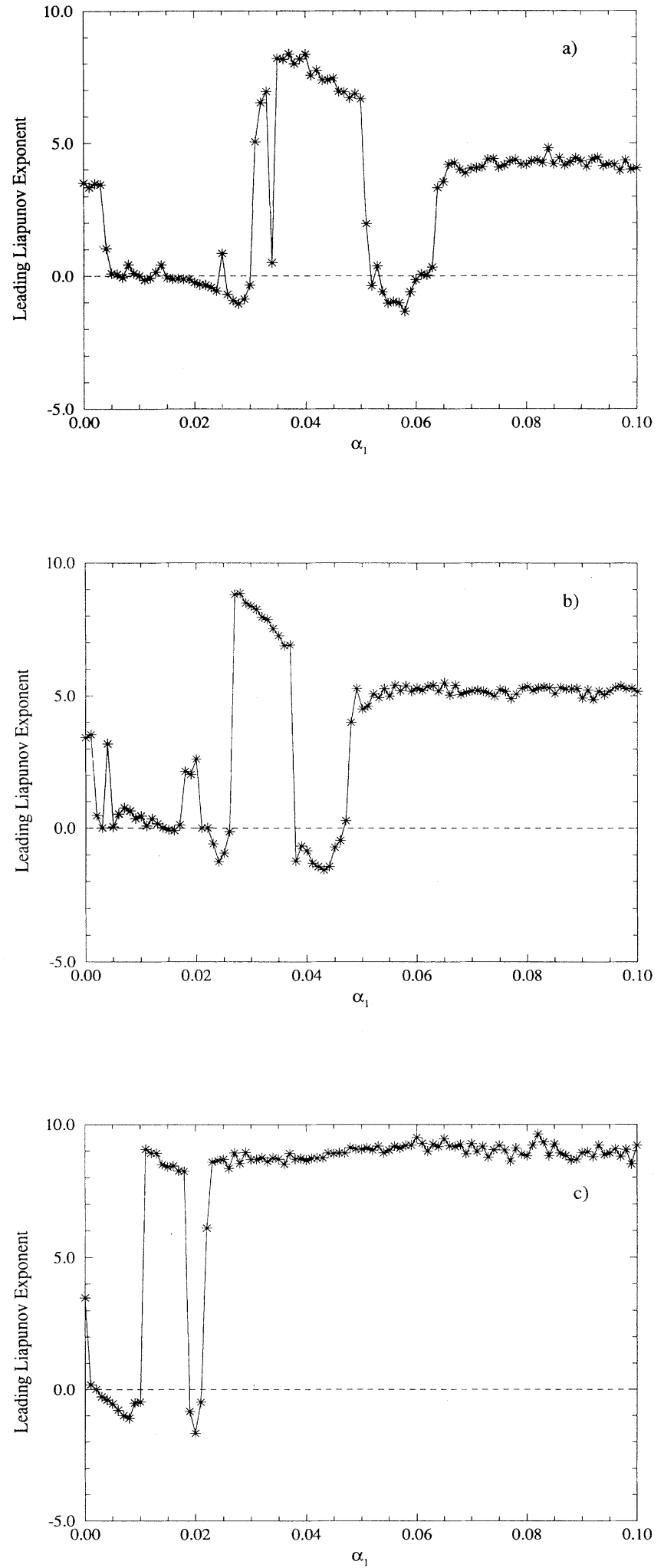

FIG. 2. Asterisks indicate the values of the leading Liapunov exponents as a function of the loss modulation amplitude $\alpha_{1}$ for different modulation frequencies. (a) $\omega=40$, (b) $\omega=50$, (c) $\omega=100$. The other parameters are as in Fig. 1. Solid lines are plotted to guide the eye.

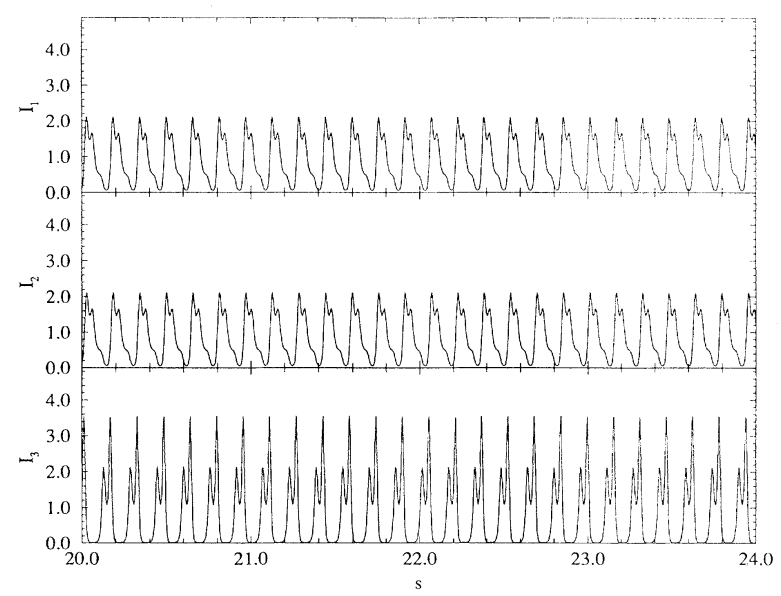

FIG. 3. Periodic time trace stabilized with $\alpha_{1}=0.025$ and $\omega=40$. The other parameters are as in Fig. 1 .

Liapunov exponent rises very fast, reaching values higher than in the absence of the modulation; thus, the amount of chaos in the system is increased. In fact we have found numerically that there are three positive Liapunov exponents in this case. The increase in the amount of chaos could be a useful feature, for example, in encoded optical communications where the output of a chaotic laser is used as a carrier [29]. In this sense, small periodic perturbations can be used as a means to maintain chaos (a method for experimental maintenance of chaos based on feedback techniques was introduced in Ref. [30]).

A further increase of the modulation amplitude leads to a drastic reduction of the leading Liapunov exponent, again reaching negative values. This region of stabilization, which was found for all three different frequencies analyzed, is characterized by a qualitatively different dynamical behavior (see Fig. 5). The resulting signal shows a periodic pulsing train with very small intensity between the peaks. These large and narrow pulses are

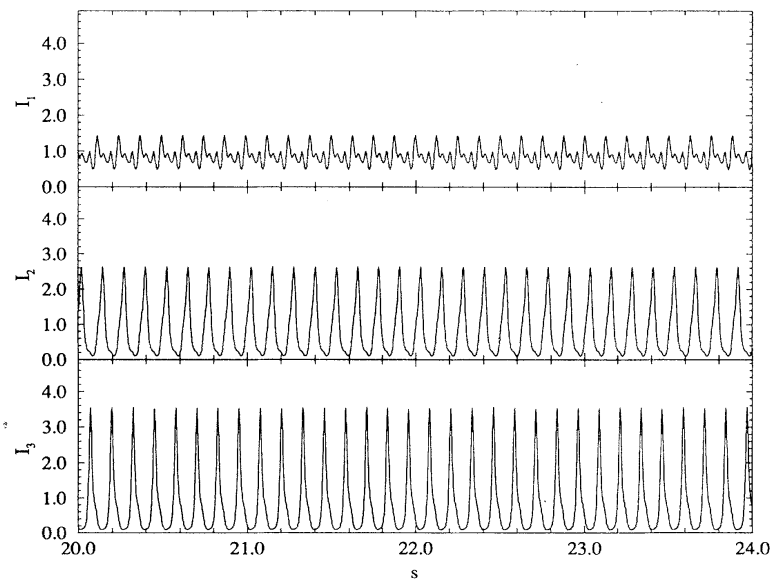

FIG. 4. Periodic time trace stabilized with $\alpha_{1}=0.006$ and $\omega=100$. The other parameters are as in Fig. 1 . 


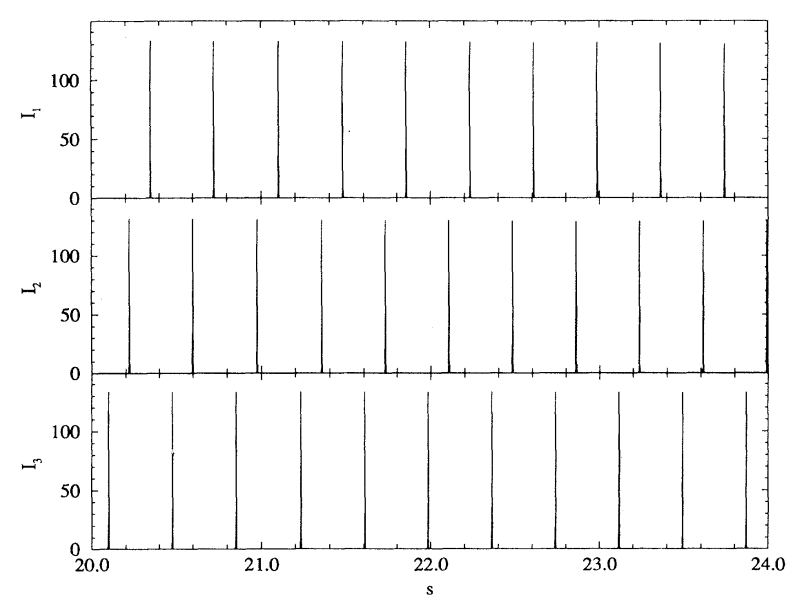

FIG. 5. Periodic time trace stabilized with $\alpha_{1}=0.043$ and $\omega=50$. The other parameters are as in Fig. 1 .

similar to those appearing in a $Q$-switching operation [24]. For the parameters of Fig. 5 the amplitude of the pulses is about 50 times higher than the total steady state intensity in the absence of the modulation. The pulses of each of the modes alternate in splay-phase behavior [31], one pulse is emitted at each period of the modulation of the losses, so that the frequency of the pulses emitted in any of the modes is $\frac{1}{3}$ of the modulation frequency.

Finally for even larger values of the modulation amplitude the leading Liapunov exponent becomes positive again. In this regime the value of all Liapunov exponents seems to be more or less constant independent of the modulation amplitude up to the largest values we have studied, $\alpha_{1}=0.1$.

We have analyzed the dependence of the leading Liapunov exponent as a function of modulation frequency for a fixed value of the amplitude (Fig. 6). Stabilization is possible within a narrow range of frequencies. This range of frequencies depends strongly on the modulation amplitude. For larger amplitude modulations, this range is located at smaller frequencies. For very small frequencies, we have not observed any stabilization.

\section{STABILIZATION USING A PERIODIC MODULATION OF THE PUMP}

In this section we analyze the case in which the laser is controlled by external modulation of the pump. Pump modulation can be used as an experimental means to study the nonlinear dynamics in the multimode Nd:YAG laser, showing (without an intracavity frequency doubling crystal) period doubling, subharmonic resonances, and harmonic resonances in far richer varieties than would be found for a single-mode laser [32]. As in the preceding section, without control the system is chaotic and we modulate the pump in the following way:

$$
p=p_{0}\left[1+p_{1} \sin (\omega s)\right] \text {. }
$$

Figure 7 shows the values of the leading Liapunov ex-
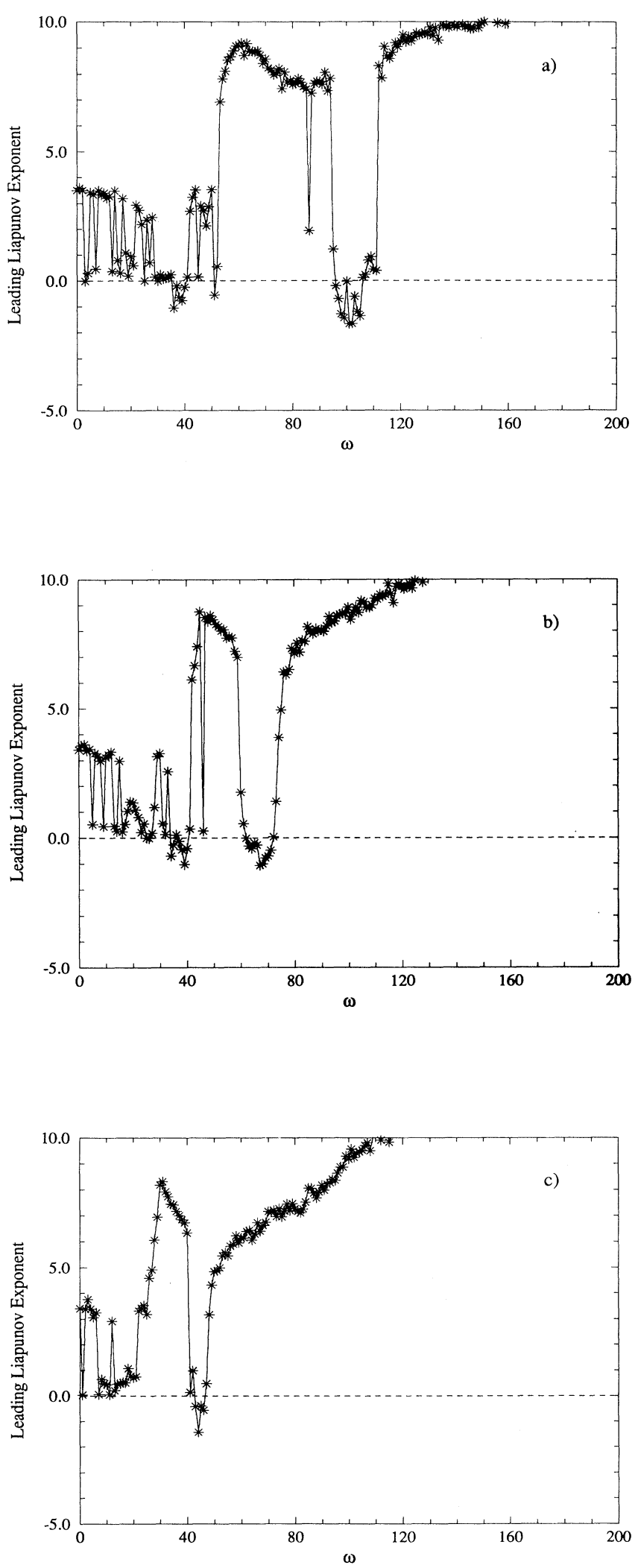

FIG. 6. Values of the leading Liapunov exponents as a function of the dimensionless loss modulation frequency $\omega$ for different modulation amplitudes. (a) $\alpha_{1}=0.02$, (b) $\alpha_{1}=0.03$, (c) $\alpha_{1}=0.05$. The other parameters are as in Fig. 1. 


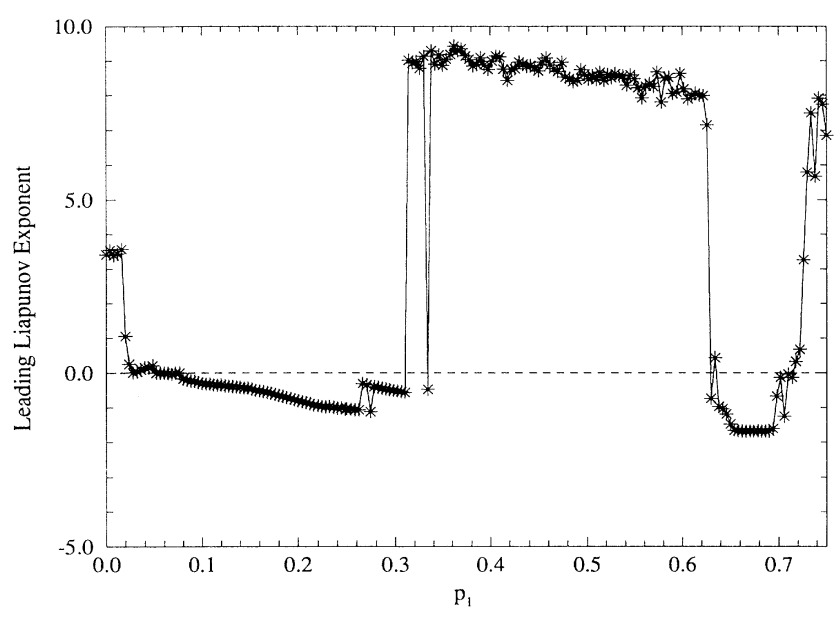

FIG. 7. Values of the leading Liapunov exponent as a function of the pump modulation amplitude $p_{1}$ for $\omega=100, g=0.1$, $\beta=0.65, p_{0}=0.03$, and $\alpha=\alpha_{0}=0.01$.

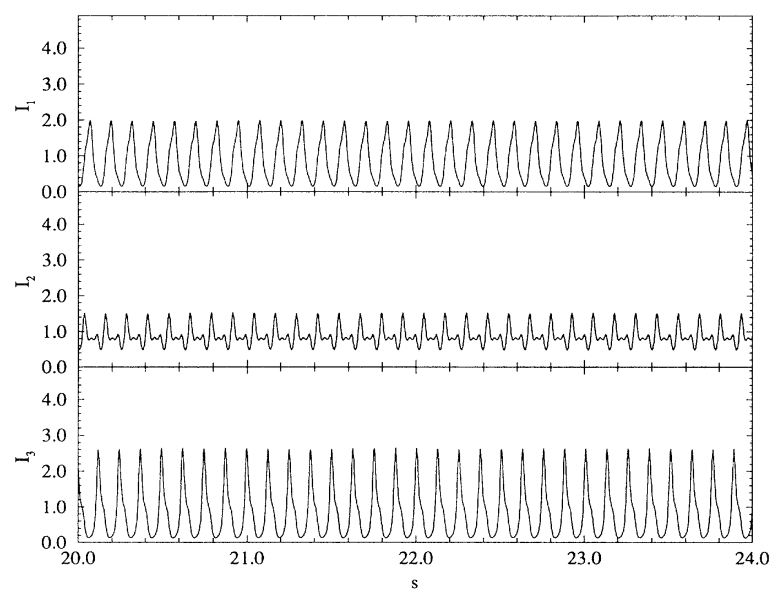

FIG. 8. Periodic time trace stabilized with $p_{1}=0.1$ and $\omega=100$. The other parameters are as in Fig. 7 .

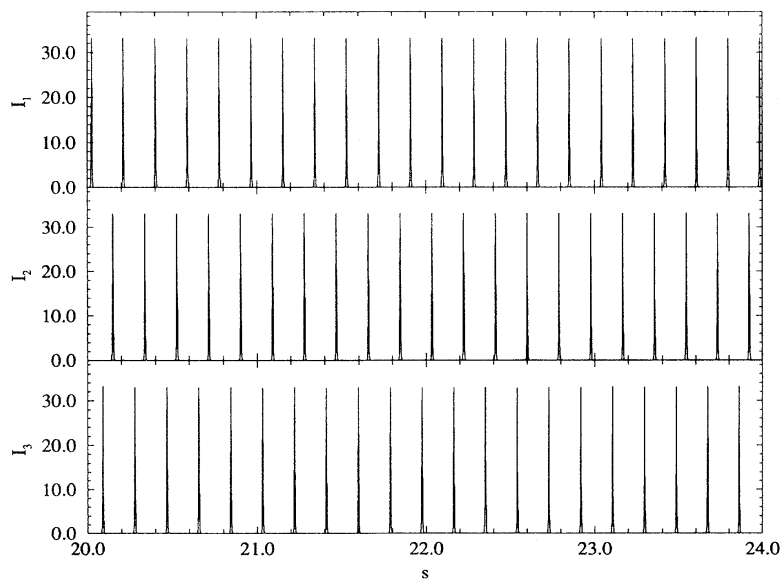

FIG. 9. Periodic time trace stabilized with $p_{1}=0.67$ and $\omega=100$. The other parameters are as in Fig. 7 . ponent as the function of pump modulation amplitude for $\omega=100\left(\Omega=416.67 \mathrm{~ms}^{-1}\right)$. In this case, if compared to the loss modulation with the same frequency [Fig. 2(c)], a larger value of the relative modulation amplitude $p_{1}$ is required in order to stabilize a periodic orbit. For given parameter values, stabilization has been obtained in two regions where the leading Liapunov exponent is negative. Figure 8 shows the time trace of the intensities of all three modes for $p_{1}=0.10$. The dynamics of the laser is periodic. Note that the periodic orbit that is obtained is qualitatively very similar to the one that was obtained by loss modulation at the same frequency and $\alpha_{1}=0.006$ (see Fig. 4). Analogous to the case of loss modulation, if the amplitude is further increased, the leading Liapunov exponent reaches very large values and the amount of chaos is increased. The further increase of the modulation amplitude (as in the case of modulation of losses) leads to a drastic reduction of the leading Liapunov exponent, again reaching negative values. This second region of stabilization is characterized by a spiky pulsed behavior, as shown in Fig. 9, with the pulses of each mode alternating in a splay-phase behavior and with one pulse emitted at each period of the modulation.

Modulating at $\omega=150$ it is possible to achieve stabilization for small amplitudes of the pump modulation, as shown in Fig. 10. Figure 11 shows the time trace obtained with a modulation amplitude $p_{1}=0.06$. It shows a pulsed behavior, though this behavior differs from the spiky case shown in Fig. 9; the pulses are smaller and the different modes have pluses of different size. Furthermore, the intensity does not drop to such small values as in the spiky case.

Figure 12 shows the value of the leading Liapunov exponent for a fixed frequency $\omega=50$ as a function of the phase difference between the loss and pump modulation. The importance of the relative phase of modulation in controlling chaos was noticed by $\mathrm{Qu}$ et al. [23]. Figure 12(a) depicts the leading Liapunov exponent as a function of the relative phase for a small value of the modulation

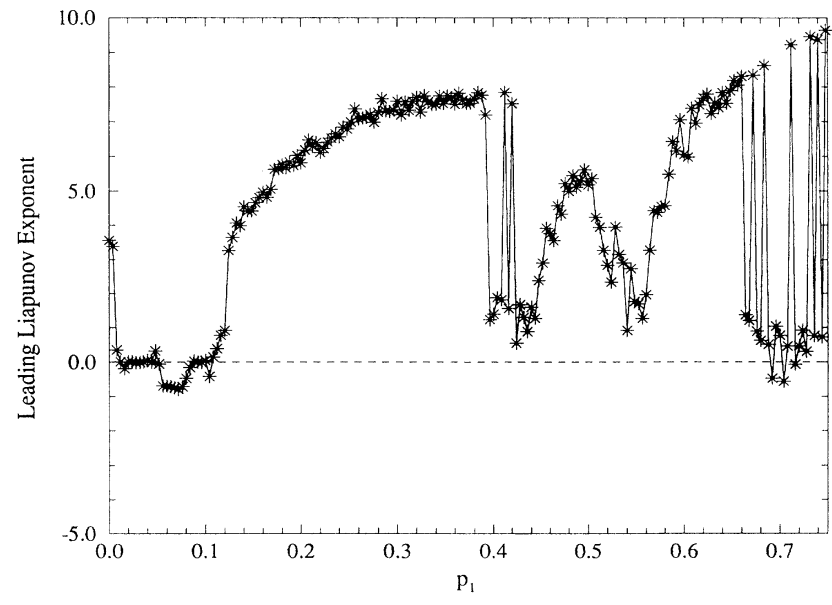

FIG. 10. Values of the leading Liapunov exponents as a function of the pump modulation amplitude $p_{1}$ for $\omega=150$. The other parameters are as in Fig. 7. 


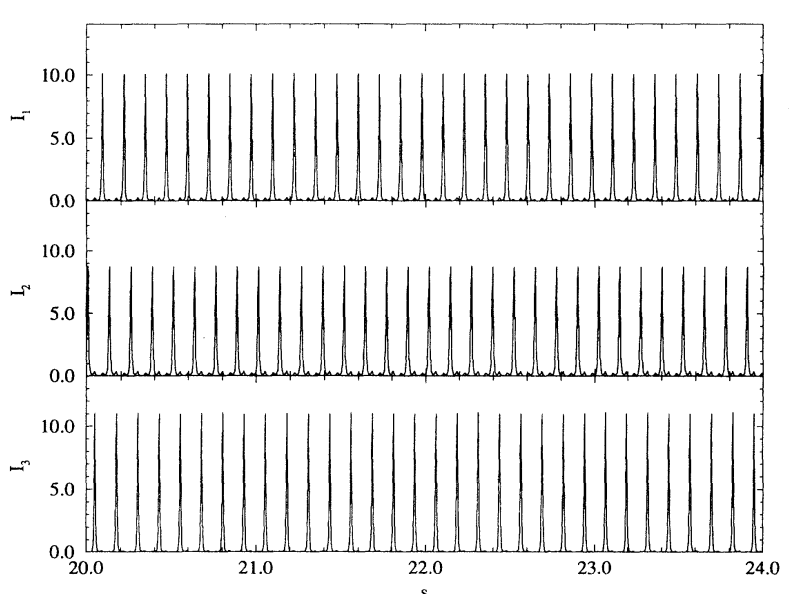

FIG. 11. Periodic time trace stabilized with $p_{1}=0.06$ and $\omega=150$. The other parameters are as in Fig. 7 .

amplitude $\alpha_{1}=p_{1}=0.01$; in this case applying only loss modulations the Liapunov exponent is reduced but still does not become negative. Figure 12(b) corresponds to the case where the leading Liapunov exponent is negative by applying only loss modulations, and Fig. 12(c) corresponds to a case where by applying only loss modulations the leading Liapunov exponent has large positive values. For these parameters, we did not observe any significant effect resulting from the constant phase between the pump and the loss modulation, except in case (b), where for some phase differences, the stabilization becomes poor.

\section{CONCLUDING REMARKS}

We have shown numerically that small periodic sinusoidal drive applied to the accessible parameters of the multimode solid state Nd:YAG laser can suppress, eliminate, or increase chaos in the system. Both parametric (losses), as well as external modulation (pump) have been studied, both leading to the same qualitative type of periodic stabilized behavior. The stabilization of the laser using modulation of losses can be reached by using very tiny amplitudes, while using modulation of the pump requires higher modulation amplitudes (for the parameter values we used). Two qualitatively different kinds of periodic orbits are obtained using either modulation of the losses or the pump. The first one is a

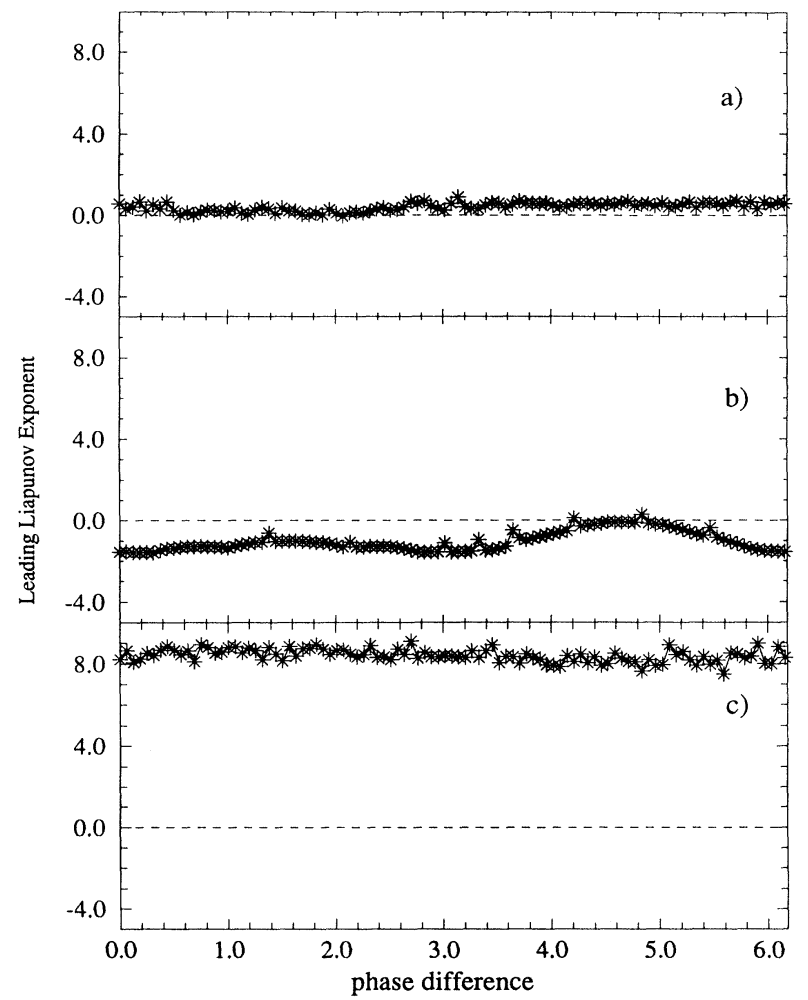

FIG. 12. Leading Liapunov exponent as a function of the phase difference between loss and gain modulation for $\omega=50$, $\alpha_{0}=0.01, \quad p_{0}=0.03$ and modulation amplitudes: (a) $\alpha_{1}=p_{1}=0.01$, (b) $\alpha_{1}=p_{1}=0.043$, (c) $\alpha_{1}=p_{1}=0.03$.

"smooth" regular pattern corresponding to periodic oscillation of each one of the modes. The second one corresponds to a pulsing train (analogous to $Q$-switching behavior). The amplitudes of the pulses are very high (about 30-100 times higher than the amplitudes of the oscillation of the uncontrolled laser), and the intensity nearly vanishes in between the pulses.

\section{ACKNOWLEDGMENTS}

P.C. was supported by Comisión Interministerial de Ciencia y Tecnología under Project No. TIC93-0744. The authors acknowledge the hospitality of Georgia Tech, where part of this work was performed. The authors thank R. Roy, K. Wiesenfeld, and N. B. Abraham for very helpful discussions and comments.
[1] S. Rajasekar and M. Lanshmanan, Physica D 67, 282 (1993); T. Shinbrot, C. Grebogi, E. Ott, and J. A. York, Nature 363, 411 (1993); E. Ott and M. Spano, Phys. Today 48, (5) (1995); J. F. Lindner and W. L. Ditto Appl. Mech. Rev. (to be published).

[2] W. L. Ditto, S. N. Rauseo, and M. L. Spano, Phys. Rev. Lett. 65, 3211 (1990); W. L. Ditto and L. M. Pecora, Sci. Am. 269, (2), 78 (1993); S. Vohra, L. Fabiny, and F. Bucholtz, Phys. Rev. Lett. 75, 65 (1995).

[3] A. Azevedo and S. M. Rezende, Phys. Rev. Lett. 66, 1342
(1991).

[4] G. Chen, IEEE Trans. Circuits Syst. I 40, 829 (1993); Int. J. Bifur. Chaos 4, 461 (1994).

[5] J. Singer, Y. Wang, and H. Bau, Phys. Rev. Lett. 66, 1123 (1991).

[6] S. Bielawski, M. Bouazaoui, D. Derozier, and P. Glorieux, Phys. Rev. A 47, 3276 (1993); B. Cipra, Science 258, 1309 (1992); A. Gavrielides, P. M. Alsing, P. M. Kovanis, and T. Erneux, Opt. Commun. 115, 551 (1995).

[7] R. Roy, T. W. Murphy, T. D. Maier, Z. Gills, and E. R. 
Hunt, Phys. Rev. Lett. 68, 1259 (1992); Z. Gills, C. Iwata, R. Roy, I. Schwartz, and I. Triandaf, ibid. 69, 3169 (1992); P. Colet, R. Roy, and K. Wiesenfeld, Phys. Rev. E 50, 3453 (1994); T. W. Carr and I. Schwartz, ibid. 51, 5109 (1995).

[8] R. Meucci, W. Gadomski, M. Ciofini, and F. T. Arecchi, Phys. Rev. E 49, R2528 (1994); N. Ciofini, R. Meucci, and F. T. Arecchi, ibid. 52, 94 (1995).

[9] D. G. Gauthier, D. W. Sukow, H. M. Concannon, and J. E. S. Socolar, Phys. Rev. E 50, 2343 (1994).

[10] B. Peng, V. Petrov, and K. Showalter, J. Phys. Chem. 95, 4957 (1991); V. Petrov, V. Gaspar, J. Masere, and K. Showalter, Nature 361, 240 (1993).

[11] M. Ye, D. E. Jones, and P. E. Wigen, J. Appl. Phys. 73, 6822 (1993).

[12] S. Hayes, C. Grebogi, E. Ott, and A. Mark, Phys. Rev. Lett. 73, 1781 (1994).

[13] V. Cimagalli, S. Jankowski, M. Giona, and T. Calascibetta, in IEEE Proceedings of the International Symposium of Circuits and Systems, Chicago IL, May 3-6, 1993, IEEE, Piscataway, NJ, 1993, pp. 2176-2179; M. Ding and J. A. S. Kelso, Proceedings of the Conference on Measuring Chaos in the Human Brain, edited by D. Duke and W. Prichard (World Scientific, Singapore, 1991), pp. 17-31; B. Doyon, B. Cessac, M. Quoy, and M. Samuelides, Int. J. Bifur. Chaos 3, 279 (1993); T. W. Frison (unpublished).

[14] A. Garfinkel, M. L. Spano, W. L. Ditto, and J. N. Weiss, Science 257, 1230 (1992).

[15] S. J. Schiff, K. Jerger, D. H. Duong, T. Chang, M. Spano, and W. L. Ditto, Nature 370, 615 (1994).

[16] E. Ott, C. Grebogi, and J. A. Yorke, Phys. Rev. Lett. 64, 1196 (1990); F. J. Romeiras, C. Grebogi, E. Ott, and W. P. Dayawansa, Physica D 58, 165 (1992); T. Tél, Int. J. Bifur. Chaos 3, 757 (1993).

[17] E. R. Hunt, Phys. Rev. Lett. 67, 1953 (1991); Z. Qu, G. Hu, and B. Ma, Phys. Lett. A 178, 265 (1993); M. A. Matías and J. Güémez, Phys. Rev. Lett. 72, 1455 (1994).

[18] K. Pyragas, Phys. Lett. A 170, 421 (1992); 181, 203 (1993); K. Pyragas and A. Tamasevicius, ibid. 180, 99 (1993).

[19] G. Nitsche and U. Dressler, Physica D 58, 153 (1991); U. Dressler and G. Nitsche, Phys. Rev. Lett. 68, 1 (1992).

[20] A. W. Hübler, Helv. Phys. Acta 62, 343 (1989); A. W. Hübler and E. Lüscher, Naturwissenschaft 76, 67 (1989);
J. L. Breeden, F. Dinkelacker, and A. W. Hübler, Phys. Rev. A 42, 5827 (1990); E. A. Jackson and A. Hübler, Physica D 44, 407 (1990); E. A. Jackson, ibid. 50, 341 (1991); Phys. Rev. A 44, 4839 (1991); R. Mettin, A. Hübler, A. Scheeline, and W. Lauterborn, Phys. Rev. E 51, 4065 (1995).

[21] R. Lima and M. Pettini, Phys. Rev. A 41, 726 (1990); L. Fronzoni, M. Giocondo, and M. Pettini, ibid. 43, 6483 (1991); F. Cuadros and R. Chacon, Phys. Rev. E 47, 4628 (1993); R. Lima and M. Pettini, ibid. 47, 4630 (1993); Y. Kivshar, F. Rodelsperger, and H. Benner, ibid. 49, 319 (1994).

[22] Y. Braiman and I. Goldhirsch, Phys. Rev. Lett. 66, 2545 (1991); M. Salerno, Phys. Rev. B 44, 2720 (1991); G. Filatrella, G. Rotoli, and M. Salterno, Phys. Lett. A 178, 81 (1993); R. Chacon and J. D. Bejarano, Phys. Rev. Lett. 71, 3103 (1993); R. Chacon, Phys. Rev. E 50, 750 (1994); G. Filatrella and G. Rotoli, Phys. Rev. B 50, 12802 (1994).

[23] Z. Qu, G. Hu, G. Yang, and G. Qin, Phys. Rev. Lett. 74, 1736 (1995).

[24] W. Koechner, Solid-State Laser Engineering (SpringerVerlag, Berlin, 1993).

[25] C. Bracikowski and R. Roy, Chaos 1, 49 (1991).

[26] D. Auerbach, C. Grebogi, E. Ott, and J. A. Yorke, Phys. Rev. Lett. 69, 3479 (1992); Hu Gang and He Kaifen, ibid. 71, 3794 (1993); J. A. Sepulchre and A. Babloyantz, Phys. Rev. E 48, 945 (1993); D. Auerbach, Phys. Rev. Lett. 72, 1184 (1994); I. Aranson, H. Levine, and L. Tsimring, ibid. 72, 2561 (1994); Y. Braiman and K. Wiesenfeld, Phys. Rev. B 49, 15223 (1994); Hu Gang and Qu Zhilin, Phys. Rev. Lett. 72, 68 (1994); G. A. Johnson, M. Lösher, and E. R. Hunt, Phys. Rev. E 51, R1625 (1995).

[27] N. Watanabe and K. Karaki, Opt. Lett. 20, 1032 (1995).

[28] Y. Liu, N. Kikuchi, and J. Ohtsubo (unpublished).

[29] P. Colet and R. Roy, Opt. Lett. 19, 2056 (1994).

[30] V. In, S. E. Mahan, W. L. Ditto, and M. Spano, Phys. Rev. Lett. 74, 4420 (1995).

[31] K. Wiesenfeld, C. Bracikowski, G. James, and R. Roy, Phys. Rev. Lett. 65, 1749 (1990); M. Silber, L. Fabiny, and K. Wiesenfeld, J. Opt. Soc. Am. B 10, 1121 (1993).

[32] N. B. Abraham, L. L. Everett, C. Iwata, and M. B. Janicki, SPIE Proc. 2095, 16 (1994). 